\title{
El uso de los árboles en Jamapa, tradiciones en un territorio deforestado
}

\section{Use of trees in Jamapa, traditions in a deforested area}

\author{
Adi Lazos-Ruíz', Patricia Moreno-Casasola²*, Sergio Guevara S.' ', Claudia Gallardo² y Eduardo Galantel
}

\author{
1 Centro Iberoamericano de la Biodiversidad (CIBIO) \\ Universidad de Alicante. San Vicente del Raspeig, \\ Alicante, España. adi_lazos@hotmail.com, galante@ \\ ua.es \\ 2 Instituto de Ecología, A.C. Red de Ecología Funcional. *Autor para correspondencia. patricia.moreno@inecol.mx \\ sergio.guevara@inecol.mx, claudia.gallardo@inecol. \\ $\mathrm{mx}$
}

\section{RESUMEN}

Los árboles han jugado un papel fundamental en el desarrollo de las civilizaciones, tanto como recursos de aprovisionamiento como por otros servicios ecosistémicos. Sin embargo hay una fuerte perturbación de los bosques tropicales que ha disminuido la cantidad y diversidad de los árboles. Una de las causas principales de esta situación es el cambio de uso del suelo, sobre todo para actividades ganaderas, que en el estado de Veracruz, México, ocupan alrededor de 3.7 millones de hectáreas (50.6\% de su territorio). El objetivo de este trabajo fue investigar el conocimiento que los ganaderos tienen sobre las especies arbóreas, sus usos, su importancia cultural y los cambios que han sucedido a lo largo del tiempo. Se mencionaron 68 especies de árboles y se hizo una clasificación de 22 tipos de usos. Se calculó el índice de importancia cultural de cada especie y se describieron los cambios más importantes en el estilo de vida de las comunidades de estudio. Se encontró que hay un conocimiento amplio sobre los árboles pero se está perdiendo rápidamente. Los árboles se usan no solo para obtener beneficios en la ganadería sino para satisfacer otras necesidades de la vida cotidiana. La cantidad y la diversidad de especies arbóreas refleja el estilo de vida de las comunidades junto con sus influencias y cambios en el tiempo. Las decisiones de los ganaderos configuran el paisaje y por lo tanto se recomienda aumentar la cantidad de árboles en los potreros, su conservación y su uso para mantener los servicios ecosistémicos que proveen.

PALAbras Clave: acciones de conservación, conocimiento local, ganadería, importancia cultural, tipos de uso.

\section{ABSTRACT}

Trees have played an important role in the development of civilizations, both for provisioning of resources and for other environmental services. However, there is a strong decrease in the quantity and diversity of tropical forests trees due to land use changes. One of the main causes of this situation is the livestock activity, which in the state of Veracruz, Mexico, occupies a surface of about 3.7 million hectares (representing $50.6 \%$ of its territory). The objective of this study was to research into cattle ranchers' knowledge of arboreal species, their uses, their cultural importance and the changes that have taken place over time. Sixty eight species of trees were mentioned and their uses were classified in 22 types of uses. The cultural importance index was calculated for each species and the most important changes in the communities' lifestyle were described. The results showed that ranchers have a wide knowledge about trees but it is rapidly disappearing. Trees are used not only to get benefits for the livestock activity but also to satisfy other needs of daily life. The quantity and diversity of arboreal species reflect the lifestyle, influences and changes over time within the communities. The decisions of ranchers configure the landscape, and therefore the recommendation is to increase the amount of trees, their conservation and uses to maintain the environmental services they provide.

KEYWORDs: conservation actions, local knowledge, livestock, cultural importance, types of use. 


\section{INTRODUCCIÓN}

Los árboles han tenido un enorme valor en el desarrollo de las civilizaciones desde tiempos preagrícolas (Casas, 2001), por sus usos con fines tanto utilitarios -madera leña, alimento y derivados medicinales- como rituales y cosmogónicos (Bellefontaine et al., 2002; López-Austin, 1997; Toledo et al., 1995). También se les confiere una gran importancia por los servicios ecosistémicos que brindan, como refugio para vida silvestre, sombra, conectividad del paisaje, regulación del clima, control de erosión, mantenimiento de biodiversidad y formación y fertilidad del suelo, entre otros (Guevara et al., 2005; Millenium Ecosystem Assessment, 2005; Moreno-Casasola y Paradowska, 2009). A pesar de su importancia, se está dando una disminución global del número de árboles de gran talla debido a causas antropogénicas (Lindenmayer et al., 2012). México es uno de los diez países con mayor cobertura de bosques primarios del mundo y es el séptimo con mayor deforestación (FAO, 2010), con una pérdida anual neta de 367224 hectáreas (Céspedes y Moreno, 2010). Esta perturbación se refleja en que más de la mitad de las superficies de bosque tropical perennifolio, subcaducifolio y caducifolio están constituidos por vegetación secundaria -i.e. la vegetación que sucede cuando se pierde el bosque original (Conafor, 2012). Los humedales arbóreos y herbáceos también han sido alterados, habiéndose perdido o degradado una superficie de 62\% (Landgrave y Moreno-Casasola, 2012). Los manglares están protegidos por la legislación ambiental mexicana, pero otros tipos de vegetación como las selvas inundables, no han sido considerados ni estudiados adecuadamente y apenas quedan bordes alrededor de los manglares (Infante et al., 2014; Landgrave y MorenoCasasola, 2012).

Las principales causas de la deforestación son el cambio de uso de suelo (Toledo, 1990), sobre todo para actividad ganadera - lo cual ha traído la proliferación de grandes extensiones de pastos introducidos para ganado bovino (Guevara y Moreno-Casasola, 2008) - y acciones promovidas por políticas públicas y programas gubernamentales en diferentes épocas del país (Bravo et al., 2010; Niembro,
2001) como el Programa Nacional de Desmontes en la década de 1970 (Moreno, 2011).

Veracruz, estado en la costa del Golfo de México, destina alrededor de 3.7 millones de hectáreas - 50.6\% de su territorio - a la ganadería (Sedarpa, 2012); es decir, los potreros son los paisajes predominantes (Guevara et al., 2005). La ganadería veracruzana se caracteriza por ser extensiva, con ganado de doble propósito (leche y carne), en unidades de pequeña escala, con un bajo nivel productivo y tecnológico y con una alta dependencia del pastizal cuya calidad y disponibilidad están marcadas por las temporadas de secas y lluvias (Travieso-Bello y MorenoCasasola, 2011; Vilaboa y Díaz, 2009).

\section{Árboles en la ganadería}

La estructura y composición arbórea de los potreros están determinadas en gran parte por las decisiones de uso y prácticas de manejo ganaderas, por ejemplo cuántos y cuáles árboles dejar en pie, cuáles sembrar o eliminar. La selección de las especies se relaciona con los beneficios que aportan para satisfacer las necesidades del ganadero (Esquivel et al., 2003; Gómez-Pompa, 1987; Gómez et al., 2013), por ejemplo para postería, cercas vivas, forraje y sombra. Los postes (troncos de aprox. $1.80 \mathrm{~m}$ de altura) son usados para sostener el alambre que delimita el espacio accesible al ganado, una hectárea necesita alrededor de 130 postes que deben ser reemplazados periódicamente. Las cercas vivas son árboles dispuestos en hilera que marcan un lindero o forman parte de las cercas con alambre; para esta finalidad usualmente se eligen especies que se propagan por estaca porque ahorran tiempo de crecimiento y evitan que sean comidas o pisadas por el ganado (Avendaño y Acosta, 2000). El forraje obtenido de hojas, tallos tiernos, flores o frutos de los árboles diversifica los recursos nutricionales para el ganado mejorando su rendimiento (Villa et al., 2009). En cuanto a la sombra de los árboles, Betancourt et al. (2003) encontraron que modifica el comportamiento de los animales en los potreros y favorece la producción de leche. Los árboles en pie dentro de los potreros no solo son proveedores de estos beneficios sino que funcionan como puntos de conectividad en el paisaje, facilitan la preserva- 
ción y dispersión de semillas y son núcleos potenciales de regeneración de las selvas (Guevara et al., 2005). De esta manera, las decisiones de los ganaderos con respecto a los árboles de sus terrenos inciden en la disponibilidad y mantenimiento de los servicios ecosistémicos.

\section{Conocimiento tradicional de los árboles}

Los principales trabajos sobre usos tradicionales de los árboles se han realizado con grupos indígenas (Toledo et al., 1995), incluyendo los mayas (Rico et al., 1991), los mixtecos (Casas et al., 1994) y los lacandones (Levy et al., 2002). Sin embargo existe poca información sobre el uso de los árboles por poblaciones mestizas - 90\% de la población rural en México (Inegi, 2010) -, y muchos menos trabajos sobre el conocimiento de los ganaderos en zonas tropicales (Muñoz, 2006). Además, actualmente hay una grave pérdida del conocimiento sobre usos tradicionales de los árboles (Reyes, 2009; González et al., 2012b). Las nuevas generaciones están cada vez menos interesadas en el campo y en general tienen poco sentido de apropiación de los recursos (Marín, 2013).

\section{OBJETIVOS}

Los objetivos de este trabajo fueron investigar cuáles especies arbóreas conocen los ganaderos, los usos que les dan, su importancia cultural y los cambios que han ocurrido en la zona a lo largo del tiempo. Se espera que los resultados de este trabajo sirvan como base para rescatar y rediseñar mejores prácticas de manejo en las zonas ganaderas, que promuevan una mayor conservación y uso de los árboles para preservar sus servicios ecosistémicos.

\section{MATERIALES Y MÉTODOS}

\section{Sitio de estudio}

El estudio se llevó a cabo en las localidades rurales de La Matamba, El Piñonal y El Yagual en el municipio de Jamapa, Veracruz, México (Fig. 1). No obstante, se considera como una sola área de estudio debido a la cercanía entre comunidades y porque todas están ubicadas en los alrededores de los últimos restos de vegetación de humedales (selva inundable) y bosque tropical caducifolio de la zona. Este lugar ha sido habitado desde tiempos prehispá-

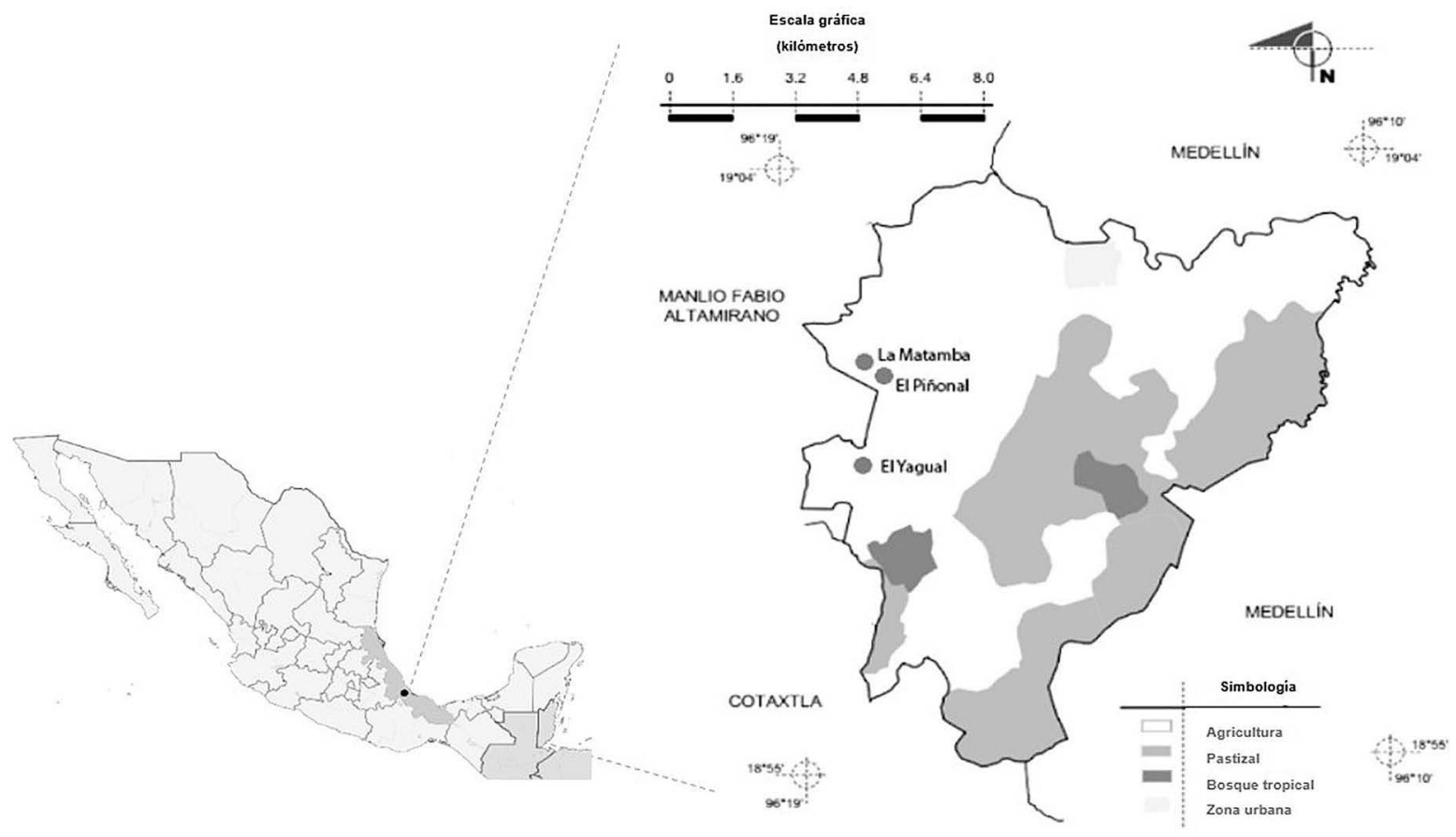

Figura 1. Ubicación, uso de suelo y vegetación del municipio de Jamapa, Veracruz, México, así como localización de las comunidades de El Piñonal, La Matamba y el Yagual (Inegi, 2009). 
nicos (Moreno-Casasola e Infante, 2009) aunque actualmente es una población mestiza; el uso tradicional de recursos prevalece hasta la actualidad pero corre el riesgo de desaparecer (González et al., 2012a y 2012b). El territorio del municipio está dedicado en $1 \%$ a zona urbana, $4 \%$ es bosque tropical (mayormente perturbado) y $95 \%$ a actividades agrícolas (i.e. cultivo de maíz y mango) y ganaderas; aunque solo 19\% de la población económicamente activa se dedica a estas actividades (Sefiplan, 2013). El clima es cálido subhúmedo con lluvias en verano, con precipitación entre $1100 \mathrm{~mm}$ y $1300 \mathrm{~mm}$ anuales, temperatura media entre $24^{\circ} \mathrm{C}$ y $26^{\circ} \mathrm{C}$ y altitud entre $10 \mathrm{~m}$ y 40 m snm (Sefiplan, 2013).

\section{Selección de informantes y entrevistas}

Se buscaron informantes dueños de terrenos ganaderos por el método de "bola de nieve", donde una persona sugiere a otra por su conocimiento y experiencia sobre el tema (Davis et al., 2010). El tamaño de la muestra se determinó por punto de redundancia y saturación de la información (Letts et al., 2007). Se llevaron a cabo entrevistas semiestructuradas (Anexo 1), habiéndose clasificado todas las respuestas (Tarrés, 2004). La información se complementó con talleres y visitas a los predios, que permitieron corroborar los resultados y enriquecer la discusión del trabajo como parte del enfoque de investigación participante (Tarrés, 2004).

\section{Colectas botánicas}

Se colectó una muestra de cada especie de árbol que los informantes mencionaron, se identificó y se depositó en el Herbario del Instituto de Ecología, A.C. (XAL). Los tipos de vegetación a los que pertenece cada especie fueron consultados en la bibliografía y en el herbario XAL, posteriormente se homologaron de acuerdo con la propuesta de Rzedowski (2006). Los nombres científicos y familias botánicas se verificaron en la base de datos Tropicos (http://www.tropicos.org/), que sigue el Grupo para la Filogenia de las Angiospermas (APG Angiosperm Phylogeny Group).

\section{Índice de Importancia Cultural}

Se calculó el Índice de Importancia Cultural (IIC) de cada especie $z$ (IIC $)$ de Turner (1988) con las modificaciones propuestas por Ávila et al. (2011) y simplificado como sigue:

$\mathrm{ICC}_{\mathrm{z}}=\frac{i u_{z}+f m_{z}+v u t_{z}}{3}$

En donde la intensidad de uso de la especie $z\left(i u_{\mathrm{z}}\right)$ se calcula con la ecuación (2), la frecuencia de mención de la especie $z$ (fmz) con la ecuación (3), el valor de la especie $z$ para el uso $\alpha\left(v u_{z \alpha}\right)$ con la ecuación (4) y el valor total de uso para la especie $z\left(v u t_{\mathrm{z}}\right)$ con la ecuación (5).

$$
\begin{aligned}
& i u_{z}=\frac{\text { Núm. de usos de la sp. } z}{\text { Núm. total de usos para todas las spp. }} \\
& f m_{z}=\frac{\text { Núm. de menciones de la sp. } z \text { para todos los usos }}{\text { Núm. total de menciones para todas las spp. para todos los usos }} \\
& v u_{z \alpha}=\frac{\text { Núm. total de menciones de la sp. } z \text { para el uso } \alpha}{\text { Núm. total de menciones para todas las spp. para el uso } \alpha} \\
& v t_{z}=\sum_{\alpha=1}^{n} v u_{z \alpha}
\end{aligned}
$$

Este índice cuantifica objetivamente la importancia de cada especie asignada por el grupo de informantes, considerando su intensidad, frecuencia y valor de uso asociados a su reconocimiento, reputación y marcaje léxico (i.e. qué tanto la gente se acuerda del nombre de la especie) a través de las menciones y los usos.

\section{RESULTADOS}

Se entrevistaron 19 personas dueñas de terrenos ganaderos: cinco mujeres y 14 hombres, de los cuales 10,5\% tenían menos de 40 años, 58\% entre 41 y 60 años y $31,5 \%$ entre 61 y 80 años. Todos se dedican a la agricultura y/o ganadería y algunos además realizan otros trabajos. Los entrevistados han tenido sus terrenos en la zona durante toda su vida, con excepción de una persona que adquirió el terreno hace menos de 10 años. Once por ciento informó que su terreno se desmontó hace menos de 10 años, $37 \%$ entre hace 20 y 40 años, $16 \%$ entre 50 y 80 años, $16 \%$ hace 100 años, $21 \%$ no supo responder. Todos 
los informantes mencionaron que su terreno se inunda en alguna época del año.

Se mencionaron 97 especies de plantas, de las cuales se excluyeron 21 por ser herbáceas y ocho por no poder identificarse al carecer de flores y frutos. Las 68 especies restantes $(70 \%$ del total) -incluyendo palmas- fueron identificadas con sus usos, las partes del árbol que se utilizan, el tipo de vegetación donde se encuentran y su valor cultural (Tabla 1).
Las familias mejor representadas fueron Fabaceae (13 especies), Moraceae (8 especies) y Malvaceae (5 especies). La tabla 2 muestra las 20 especies mencionadas por más informantes, las especies con mayor número de usos, las especies de las que se utiliza un mayor número de partes y las especies con valor cultural más alto. Gliricidia sepium y Maclura tinctoria aparecen entre los primeros cinco lugares de todas las categorías.

TABLA 1. Especies de árboles mencionadas y sus usos.

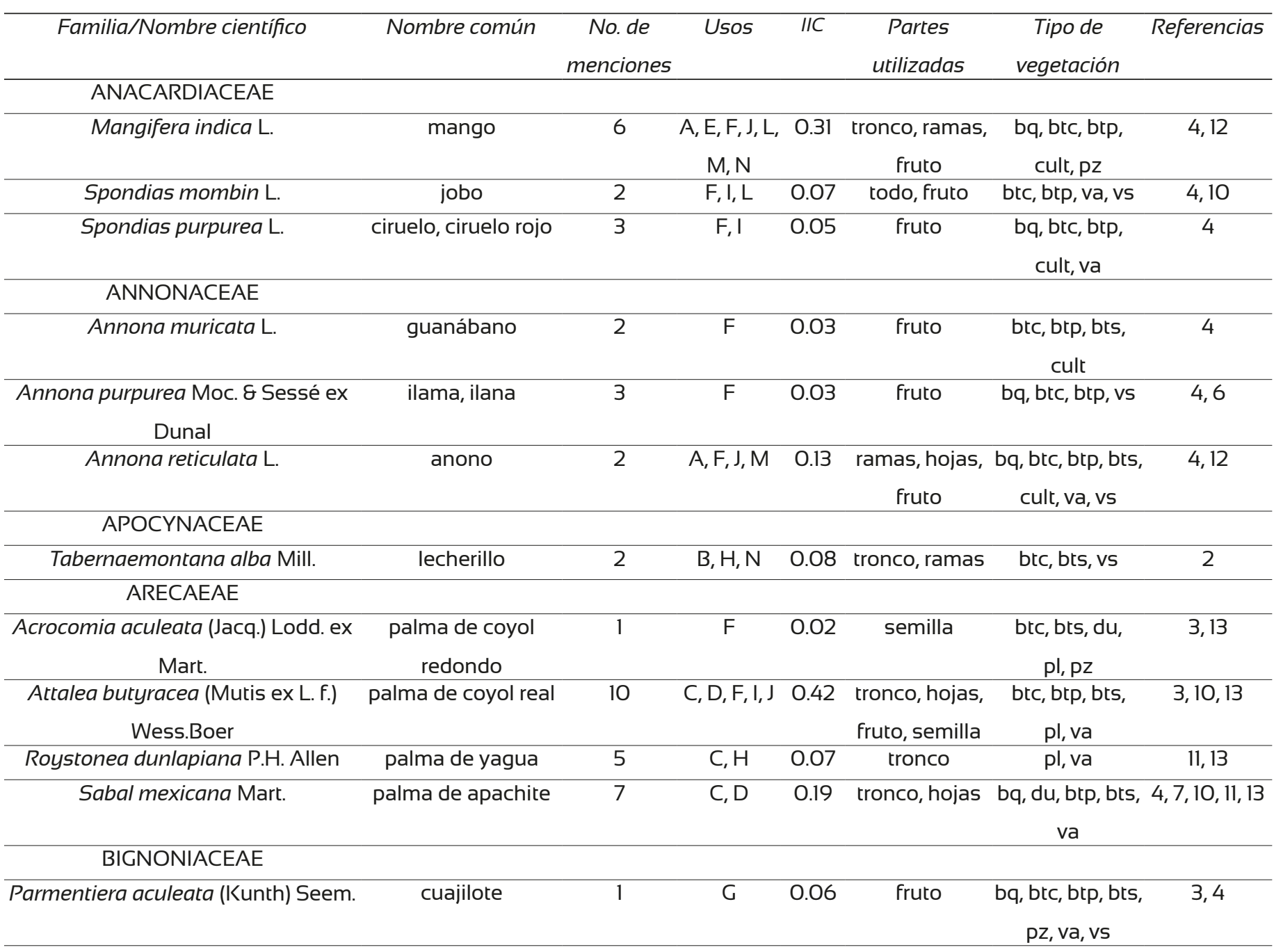

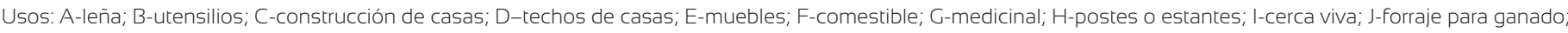
K-ornamental; L-sombra; M-vida silvestre; N-madera; O-conservación; P-artesanías; Q-ritual; R-cortina rompevientos; S-otros; T-tinta.

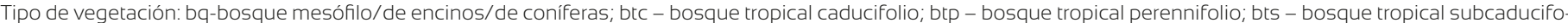
lio; cult-cultivada; du-dunas; pl-palmar; pz-pastizal; va - vegetación acuática (incluye selva inundable/ripario/manglares/esteros); vs-vegetación secundaria.

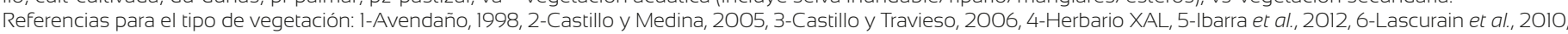

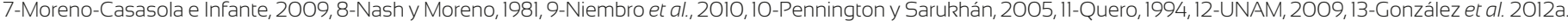


TABla 1. Especies de árboles mencionadas y sus usos. (Continuación...)

\begin{tabular}{|c|c|c|c|c|c|c|c|}
\hline Familia/Nombre científico & Nombre común & $\begin{array}{c}\text { No. de } \\
\text { menciones }\end{array}$ & Usos & IIC & $\begin{array}{c}\text { Partes } \\
\text { utilizadas }\end{array}$ & $\begin{array}{c}\text { Tipo de } \\
\text { vegetación }\end{array}$ & Referencias \\
\hline Roseodendron donnell-smithii (Rose) & primavera & 1 & I & 0.02 & todo & btc, bts & 10 \\
\hline \multicolumn{8}{|l|}{ Miranda } \\
\hline Tabebuia rosea (Bertol.) DC. & roble & 9 & $\begin{array}{l}A, B, C, E \\
H, I, L, N\end{array}$ & 0.46 & $\begin{array}{l}\text { todo, tronco, } \\
\text { ramas }\end{array}$ & $\begin{array}{l}\text { btc, btp, bts, du, } \\
\text { pz, va, vs }\end{array}$ & 3,4 \\
\hline \multicolumn{8}{|l|}{ BORAGINACEAE } \\
\hline Cordia cf. diversifolia Pav. ex DC. & tepozán & 1 & G & 0.03 & hojas & btc, bts & 3 \\
\hline Cordia collococca L. & nopo & 3 & $\mathrm{C}, \mathrm{I}$ & 0.06 & tronco & bq, btp, bts, va & 8 \\
\hline Cordia dodecandra DC. & cópite & 7 & $\begin{array}{c}B, C, F, I, J \\
M, N\end{array}$ & 0.28 & $\begin{array}{l}\text { todo, tronco, } \\
\text { ramas, hojas, } \\
\text { fruto }\end{array}$ & btc, cult, vs & 4,10 \\
\hline Ehretia tinifolia L. & frutillo, rayado & 4 & $\begin{array}{c}A, L, M \\
O, S\end{array}$ & 0.53 & todo & $\begin{array}{l}\text { bq, btc, btp, } \\
\text { bts, vs }\end{array}$ & $4,8,9$ \\
\hline \multicolumn{8}{|l|}{ BURSERACEAE } \\
\hline Bursera simaruba (L.) Sarg. & mulato, palo mulato & 10 & G, I, L, M & 0.18 & $\begin{array}{l}\text { tronco, ramas, } \\
\text { hojas, corteza }\end{array}$ & $\begin{array}{l}\text { bq, btc, btp, bts, } \\
\text { du, pz, va, vs }\end{array}$ & $3,4,10$ \\
\hline \multicolumn{8}{|l|}{ CASUARINACEAE } \\
\hline Casuarina equisetifolia $\mathrm{L}$. & pino & 1 & $\mathrm{R}$ & 0.18 & todo & $\begin{array}{l}\text { bq, btc, btp, } \\
\text { cult, du, va, vs }\end{array}$ & 4 \\
\hline \multicolumn{8}{|l|}{ COMBRETACEAE } \\
\hline Terminalia catappa L. & almendro & 1 & $\mathrm{~F}, \mathrm{~L}$ & 0.04 & todo, semilla & cult & - \\
\hline \multicolumn{8}{|l|}{ EBENACEAE } \\
\hline Diospyros nigra (J.F. Gmel.) Perr. & $\begin{array}{l}\text { zapote negro, } \\
\text { zapote prieto }\end{array}$ & 6 & $\mathrm{~F}$ & 0.04 & fruto & $\begin{array}{l}\text { bq, btc, btp, bts, } \\
\text { cult, va }\end{array}$ & 6,10 \\
\hline \multicolumn{8}{|l|}{ FABACEAE } \\
\hline $\begin{array}{c}\text { Acacia cochliacantha Humb. \& } \\
\text { Bonpl. ex Willd. }\end{array}$ & huizache & 6 & $A, C, H, I, J$ & 0.25 & $\begin{array}{l}\text { tronco, ramas, } \\
\text { fruto }\end{array}$ & $\begin{array}{l}\text { bq, btc, bts, } \\
\text { dun, pz, va, vs }\end{array}$ & 4 \\
\hline Caesalpinia cacalaco Bonpl. & tihuil & 7 & $\mathrm{~A}, \mathrm{H}, \mathrm{I}, \mathrm{N}$ & 0.12 & $\begin{array}{l}\text { todo, tronco, } \\
\text { ramas }\end{array}$ & btc & 9 \\
\hline Cassia fistula L. & lluvia de oro & 1 & $\mathrm{~K}, \mathrm{~L}$ & 0.37 & todo & btc, bts, cult, vs. & 4 \\
\hline Diphysa robinioides Benth. & amarillo, quebrache & 7 & $\begin{array}{c}\mathrm{B}, \mathrm{H}, \mathrm{I}, \mathrm{L} \\
\mathrm{N}, \mathrm{T}\end{array}$ & 0.35 & $\begin{array}{l}\text { todo, tronco, } \\
\text { ramas, corazón }\end{array}$ & $\begin{array}{l}\text { btc, btp, bts, du, } \\
\text { va, vs }\end{array}$ & 4,10 \\
\hline $\begin{array}{l}\text { Enterolobium cyclocarpum (Jacq.) } \\
\text { Griseb. }\end{array}$ & $\begin{array}{l}\text { nacaxtle, nacastle, } \\
\text { nacaste }\end{array}$ & 4 & $\begin{array}{l}\text { A, E, F, } \\
L, N\end{array}$ & 0.14 & $\begin{array}{l}\text { tronco, ramas, } \\
\text { fruto }\end{array}$ & $\begin{array}{l}\text { bq, btc, btp, bts, } \\
\text { du, pz, va, vs }\end{array}$ & $3,4,10$ \\
\hline $\begin{array}{l}\text { Gliricidia sepium (Jacq.) Kunth ex } \\
\text { Walp. }\end{array}$ & cocuite & 12 & $\begin{array}{c}A, B, C, F \\
H, I, J, L \\
N, S\end{array}$ & 0.79 & $\begin{array}{l}\text { todo, tronco, } \\
\text { ramas, flor, } \\
\text { hojas, brotes }\end{array}$ & $\begin{array}{l}\text { bq, btc, btp, bts, } \\
\text { du, pz, va, vs }\end{array}$ & 4,10 \\
\hline Haematoxylum campechianum L. & tinto & 2 & $\mathrm{~B}, \mathrm{C}, \mathrm{H}, \mathrm{I}, \mathrm{T}$ & 0.24 & $\begin{array}{l}\text { tronco, ramas, } \\
\text { corazón }\end{array}$ & $\begin{array}{l}\text { btc, btp, } \mathrm{pz}, \\
\text { va, vs }\end{array}$ & 4 \\
\hline Inga sp. & chalahuite & 1 & G & 0.03 & corteza & - & - \\
\hline Leucaena leucocephala (Lam.) de Wit & guaje & 2 & $\mathrm{~F}, \mathrm{G}$ & 0.05 & corteza, fruto & $\begin{array}{l}\text { bq, btc, btp, bts, } \\
\text { du, va }\end{array}$ & 4 \\
\hline Lonchocarpus sp. & marinero & 5 & A, C, L & 0.12 & todo, ramas & - & - \\
\hline
\end{tabular}


TABLA 1. Especies de árboles mencionadas y sus usos. (Continuación...)

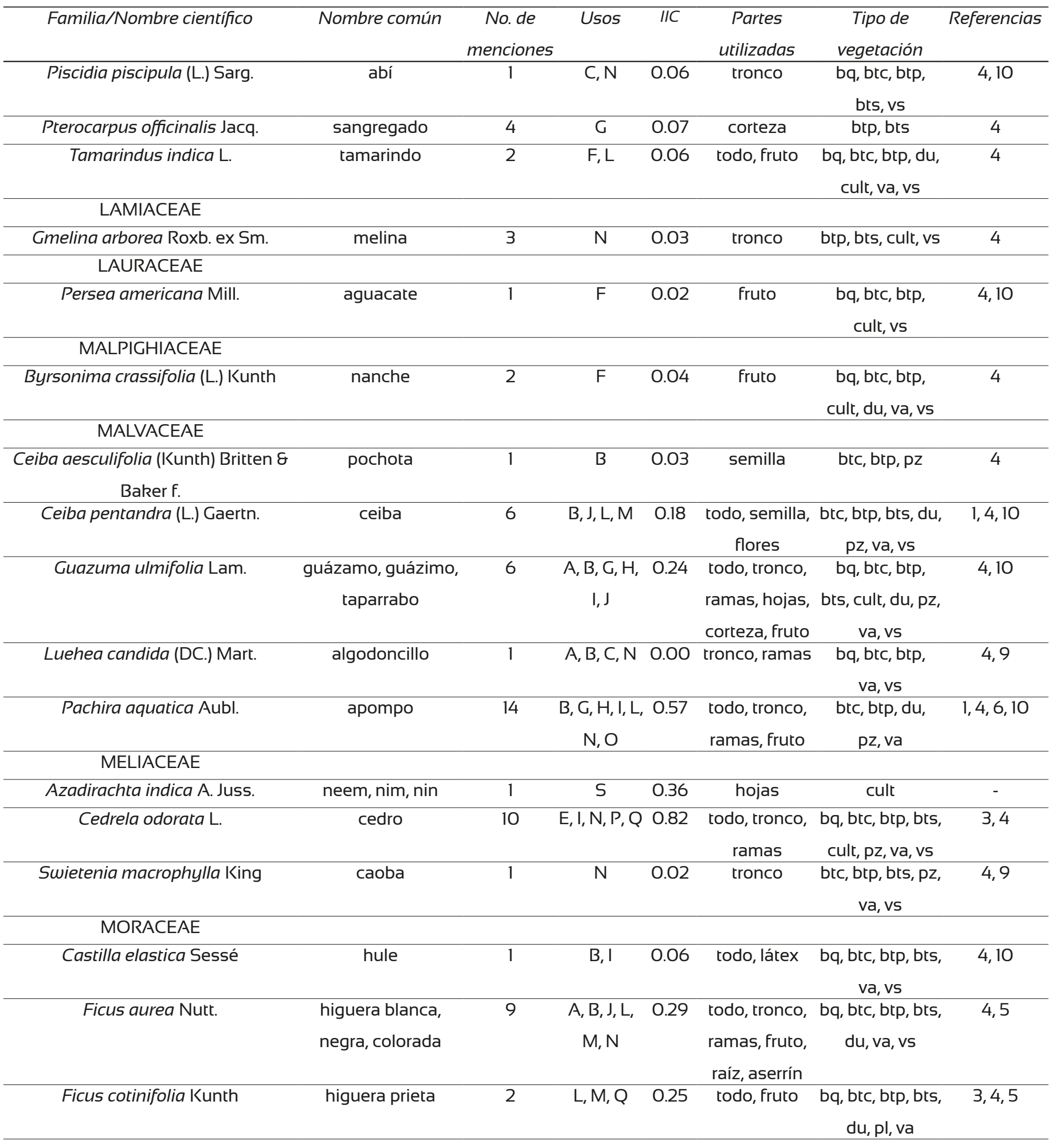

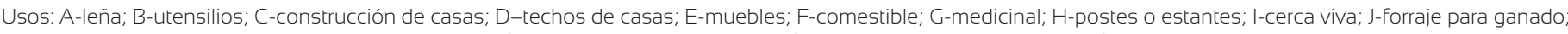
K-ornamental; L-sombra; M-vida silvestre; N-madera; O-conservación; P-artesanías; Q-ritual; R-cortina rompevientos; S-otros; T-tinta.

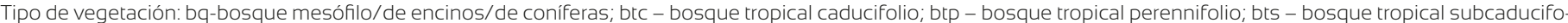
lio; cult-cultivada; du-dunas; pl-palmar; pz-pastizal; va - vegetación acuática (incluye selva inundable/ripario/manglares/esteros); vs-vegetación secundaria.

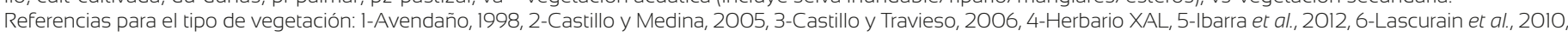

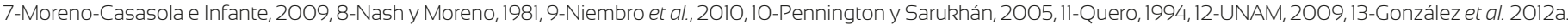


TAвLA 1. Especies de árboles mencionadas y sus usos. (Continuación...)

\begin{tabular}{|c|c|c|c|c|c|c|c|}
\hline Familia/Nombre científico & Nombre común & $\begin{array}{c}\text { No. de } \\
\text { menciones }\end{array}$ & Usos & IIC & $\begin{array}{c}\text { Partes } \\
\text { utilizadas }\end{array}$ & $\begin{array}{c}\text { Tipo de } \\
\text { vegetación }\end{array}$ & Referencias \\
\hline Ficus crocata (Miq.) Miq. & $\begin{array}{c}\text { higuera negra, } \\
\text { higuera de tendón }\end{array}$ & 7 & A, L, M & 0.13 & $\begin{array}{l}\text { todo, tronco, } \\
\text { ramas, raíz }\end{array}$ & $\begin{array}{l}\text { bq, btc, btp, bts, } \\
\text { du, pz, va, vs }\end{array}$ & 4,5 \\
\hline Ficus obtusifolia Kunth & hule & 2 & B & 0.03 & látex & $\begin{array}{l}\text { bq, btc, btp, bts, } \\
\text { du, pl, va, vs }\end{array}$ & 2 \\
\hline Ficus sp. & higuera & 3 & $\mathrm{O}$ & 0.10 & todo & & \\
\hline Ficus yoponensis Desv. & higuera blanca & 1 & $J, R$ & 0.21 & todo, fruto & $\begin{array}{l}\text { bq, btp, bts, pz, } \\
\text { va, vs }\end{array}$ & 4.5 \\
\hline $\begin{array}{c}\text { Maclura tinctoria (L.) D. Don ex } \\
\text { Steud. }\end{array}$ & moral, mora & 10 & $\begin{array}{c}A, B, C, F, \\
H, I, J, L \\
M, T\end{array}$ & 0.47 & $\begin{array}{l}\text { todo, tronco, } \\
\text { ramas, fruto, } \\
\text { corazón }\end{array}$ & $\begin{array}{l}\text { bq, btc, btp, bts, } \\
\text { du, pz, va, vs }\end{array}$ & $3,4,10$ \\
\hline \multicolumn{8}{|l|}{ MUNTINGIACEAE } \\
\hline Muntingia calabura L. & capulín, nigüilla & 1 & $\mathrm{~F}$ & 0.02 & fruto & $\begin{array}{l}\text { bq, btc, btp, bts, } \\
\text { cult, du, pz, va, vs }\end{array}$ & 4 \\
\hline \multicolumn{8}{|l|}{ MYRTACEAE } \\
\hline Psidium guajava $\mathrm{L}$. & guayaba & 2 & $F, G$ & 0.05 & hojas, fruto & $\begin{array}{c}\text { bq, btc, btp, } \\
\text { bts, cult, du, pz, } \\
\text { va, vs }\end{array}$ & $3,4,6,10$ \\
\hline \multicolumn{8}{|l|}{ POLYGONACEAE } \\
\hline Coccoloba barbadensis Jacq. & uvero & 1 & I & 0.02 & todo & $\begin{array}{l}\text { bq, btc, btp, bts, } \\
\text { du, pl, pz, va, vs }\end{array}$ & $3,4,10$ \\
\hline \multicolumn{8}{|l|}{ PRIMULACEAE } \\
\hline Ardisia sp. & capulín & 1 & $\mathrm{~F}$ & 0.02 & fruto & - & - \\
\hline \multicolumn{8}{|l|}{ RUBIACEAE } \\
\hline Genipa americana L. & yual & 3 & $\mathrm{~F}$ & 0.03 & fruto & $\begin{array}{l}\text { bq, btc, btp, bts, } \\
\text { cult, pz, va, vs }\end{array}$ & 3,4 \\
\hline Randia sp. & crucetillo & 2 & G, M & 0.08 & fruto & - & - \\
\hline \multicolumn{8}{|l|}{ RUTACEAE } \\
\hline Citrus limon (L.) Osbeck & limón & 1 & $\mathrm{~F}$ & 0.02 & fruto & cult & - \\
\hline Citrus sinensis (L.) Osbeck & naranjo & 2 & $\mathrm{~F}$ & 0.03 & fruto, hojas & cult & - \\
\hline \multicolumn{8}{|l|}{ SALICACEAE } \\
\hline $\begin{array}{c}\text { Pleuranthodendron lindenii (Turcz.) } \\
\text { Sleumer }\end{array}$ & catarrito & 1 & A & 0.03 & tronco, ramas & $\begin{array}{l}\text { bq, btc, btp, bts, } \\
\text { va, vs }\end{array}$ & $3,4,10$ \\
\hline Salix humboldtiana Willd. & sauce & 7 & $\begin{array}{c}\mathrm{B}, \mathrm{E}, \mathrm{H}, \mathrm{L}, \\
\mathrm{N}, \mathrm{O}\end{array}$ & 0.26 & $\begin{array}{l}\text { todo, tronco, } \\
\text { ramas, hojas }\end{array}$ & $\begin{array}{l}\text { bq, btc, btp, bts, } \\
\text { pz, va, vs }\end{array}$ & $4,7,10$ \\
\hline $\begin{array}{l}\text { Zuelania guidonia (Sw.) Britton \& } \\
\text { Millsp. }\end{array}$ & palo volador, volador & 2 & $\mathrm{C}, \mathrm{H}$ & 0.08 & tronco & $\begin{array}{l}\text { bq, btc, btp, pz, } \\
\text { va, vs }\end{array}$ & 4 \\
\hline \multicolumn{8}{|l|}{ SAPINDACEAE } \\
\hline Melicoccus oliviformis Kunth & guaya & 1 & $\mathrm{~F}$ & 0.02 & fruto & btc, btp & 6 \\
\hline \multicolumn{8}{|l|}{ SAPOTACEAE } \\
\hline Manilkara zapota (L.) P. Royen & $\begin{array}{l}\text { zapote chico, chico- } \\
\text { zapote }\end{array}$ & 2 & $F, L, M$ & 0.09 & fruto, látex & $\begin{array}{l}\text { btc, btp, bts, } \\
\text { cult, du, pz, } \\
\text { va, vs }\end{array}$ & 4,9 \\
\hline
\end{tabular}


TABla 1. Especies de árboles mencionadas y sus usos. (Final).

\begin{tabular}{|c|c|c|c|c|c|c|c|}
\hline Familia/Nombre científico & Nombre común & $\begin{array}{c}\text { No. de } \\
\text { menciones }\end{array}$ & Usos & IIC & $\begin{array}{c}\text { Partes } \\
\text { utilizadas }\end{array}$ & $\begin{array}{c}\text { Tipo de } \\
\text { vegetación }\end{array}$ & Referencias \\
\hline \multicolumn{8}{|l|}{ URTICACEAE } \\
\hline Cecropia obtusifolia Bertol. & chancarro, guarumo & 3 & G & 0.06 & hojas & $\begin{array}{c}\text { bq, btc, btp, } \\
\text { va, vs }\end{array}$ & 4,10 \\
\hline
\end{tabular}

Usos: A-leña; B-utensilios; C-construcción de casas; D-techos de casas; E-muebles; F-comestible; G-medicinal; H-postes o estantes; I-cerca viva; J-forraje para ganado; K-ornamental; L-sombra; M-vida silvestre; N-madera; O-conservación; P-artesanías; Q-ritual; R-cortina rompevientos; S-otros; T-tinta.

Tipo de vegetación: bq-bosque mesófilo/de encinos/de coníferas; btc - bosque tropical caducifolio; btp - bosque tropical perennifolio; bts - bosque tropical subcaducifolio; cult-cultivada; du-dunas; pl-palmar; pz-pastizal; va - vegetación acuática (incluye selva inundable/ripario/manglares/esteros); vs-vegetación secundaria.

Referencias para el tipo de vegetación: 1-Avendaño, 1998, 2-Castillo y Medina, 2005, 3-Castillo y Travieso, 2006, 4-Herbario XAL, 5-Ibarra et al., 2012, 6-Lascurain et al., 2010, 7-Moreno-Casasola e Infante, 2009, 8-Nash y Moreno, 1981, 9-Niembro et al., 2010, 10-Pennington y Sarukhán, 2005, 11-Quero, 1994, 12-UNAM, 2009, 13-González et al. 2012a.

Las especies pueden pertenecer a más de un tipo de vegetación. Sesenta y tres porciento de todas las especies ocurren en el bosque tropical subcaducifolio, $54 \%$ en el bosque tropical caducifolio y $40 \%$ en el bosque tropical perennifolio; $62 \%$ en zonas inundables; $60 \%$ como vegetación secundaria y $32 \%$ son cultivadas. Las especies que ocurren en más tipos de vegetación son Bursera simaruba, Coccoloba barbadensis, Enterolobium cyclocarpum, Guazuma ulmifolia, Maclura tinctoria, Manilkara zapota, Muntingia calabura y Psidium guajava. Muchas de estas especies también son comunes en vegetación secundaria o acahuales.

Se identificaron 22 usos distintos de los árboles, que fueron clasificados en tres grupos: uso maderable, uso extractivo y uso no extractivo. Los usos maderables requieren el tronco entero, lo que provoca que se elimine completamente el árbol; los usos extractivos usan alguna parte del árbol permitiendo que se regenere y los usos no extractivos son los beneficios que se obtienen del árbol completo y vivo, lo que le permite seguir su desarrollo natural. La tabla 3 muestra los tipos de uso y el número de especies registradas para cada uno. Los usos con una mayor diversidad de especies son el comestible (26 especies), sombra (20 especies), cerca viva (19 especies) y madera (16 especies); mientras que para los usos como artesanías, insecticida y ornamental se mencionó solo una especie para cada uno. Del total de usos, los maderables representan $18 \%$, los extractivos $46 \%$ y los no extractivos $36 \%$.

\section{Usos maderables}

Las especies con más usos de la categoría maderable y más mencionadas fueron Tabebuia rosea, Cedrela odorata, Diphysa robinioides y Mangifera indica. La tabla 4 muestra los usos y las características de algunas especies maderables. Para la construcción de muebles se requieren maderas finas y fuertes como las de Cedrela odorata y Tabebuia rosea. Los árboles con fustes largos y derechos como Zuelania guidonia casi han desaparecido por completo. La producción de tinturas está en esta clasificación puesto que se utiliza el duramen de los árboles, es decir requiere troncos maduros, por ejemplo de Diphysa robinioides, Haematoxylum campechianum y Maclura tinctoria. Aunque en las comunidades no les dan este uso, los informantes lo refirieron.

\section{Usos extractivos}

Las especies más conocidas por el mayor número de usos extractivos y que fueron más mencionadas son Gliricidia sepium, Maclura tinctoria, Guazuma ulmifolia y Pachira aquatica. Para elaboración de artesanías se refirieron únicamente a las semillas de Cedrela odorata; sin embargo se observó que un grupo de artesanas de La Matamba elaboraba productos con semillas de Cocos nucifera y Acrocomia aculeata. Para el uso comestible se aprovechan los frutos (mesocarpo) de todas las especies citadas excepto de Gliricidia sepium, de la que se come la flor, y de las palmas Acrocomia aculeata y Attalea butyracea, de las que se come el endospermo de la semilla. Para el uso como 
TAвLA 2. Las veinte especies arbóreas con más menciones, usos, partes utilizadas e importancia cultural.

\begin{tabular}{|c|c|c|c|c|c|c|c|c|}
\hline Lugar & $\begin{array}{c}\text { Especies con más } \\
\text { menciones }\end{array}$ & $\begin{array}{c}\text { No. de } \\
\text { menciones }\end{array}$ & $\begin{array}{c}\text { Especies con más } \\
\text { usos }\end{array}$ & $\begin{array}{l}\text { No. de } \\
\text { usos }\end{array}$ & $\begin{array}{l}\text { Especies con más } \\
\text { partes utilizadas }\end{array}$ & $\begin{array}{l}\text { No. de } \\
\text { partes }\end{array}$ & $\begin{array}{c}\text { Especies con IIC más } \\
\text { alto }\end{array}$ & IIC \\
\hline $1^{\circ}$ & Pachira aquatica & 14 & Gliricidia sepium & 11 & Gliricidia sepium & 6 & Cedrela odorata & 0.82 \\
\hline $2^{\circ}$ & Gliricidia sepium & 12 & Maclura tinctoria & 10 & Ficus aurea & 6 & Gliricidia sepium & 0.79 \\
\hline $3^{\circ}$ & Maclura tinctoria & 10 & Tabebuia rosea & 8 & Guazuma ulmifolia & 6 & Pachira aquatica & 0.57 \\
\hline $4^{\circ}$ & Cedrela odorata & 10 & Pachira aquatica & 7 & Maclura tinctoria & 5 & Ehretia tinifolia & 0.53 \\
\hline $5^{\circ}$ & Attalea butyracea & 10 & Cordia dodecandra & 7 & Cordia dodecandra & 5 & Maclura tinctoria & 0.47 \\
\hline $6^{\circ}$ & Bursera simaruba & 10 & Mangifera indica & 7 & Pachira aquatica & 4 & Tabebuia rosea & 0.46 \\
\hline $7^{\circ}$ & Tabebuia rosea & 9 & Ficus aurea & 6 & Diphysa robinioides & 4 & Attalea butyracea & 0.42 \\
\hline $8^{\circ}$ & Ficus aurea & 9 & Diphysa robinioides & 6 & Salix humboldtiana & 4 & Cassia fistula & 0.37 \\
\hline $9^{\circ}$ & Cordia dodecandra & 7 & Salix humboldtiana & 6 & Attalea butyracea & 4 & Azadirachta indica & 0.36 \\
\hline $10^{\circ}$ & Diphysa robinioides & 7 & Guazuma ulmifolia & 6 & Bursera simaruba & 4 & Diphysa robinioides & 0.35 \\
\hline $11^{\circ}$ & Salix humboldtiana & 7 & Cedrela odorata & 5 & Ficus crocata & 4 & Mangifera indica & 0.31 \\
\hline $12^{\circ}$ & Caesalpinia cacalaco & 7 & Attalea butyracea & 5 & Tabebuia rosea & 3 & Ficus aurea & 0.29 \\
\hline $13^{\circ}$ & Ficus crocata & 7 & Acacia cochliacantha & 5 & Mangifera indica & 3 & Cordia dodecandra & 0.28 \\
\hline $14^{\circ}$ & Sabal mexicana & 7 & Ceiba pentandra & 5 & Cedrela odorata & 3 & Salix humboldtiana & 0.26 \\
\hline $15^{\circ}$ & Mangifera indica & 6 & Enterolobium & 5 & Acacia cochliacantha & 3 & Acacia cochliacantha & 0.25 \\
\hline $16^{\circ}$ & Guazuma ulmifolia & 6 & $\begin{array}{l}\text { cyclocarpum } \\
\text { Haematoxylum } \\
\text { campechianum }\end{array}$ & 5 & Ceiba pentandra & 3 & Ficus cotinifolia & 0.25 \\
\hline $17^{\circ}$ & Acacia cochliacantha & 6 & Bursera simaruba & 4 & $\begin{array}{l}\text { Enterolobium cyclo- } \\
\text { carpum }\end{array}$ & 3 & $\begin{array}{l}\text { Haematoxylum } \\
\text { campechianum }\end{array}$ & 0.24 \\
\hline $18^{\circ}$ & Ceiba pentandra & 6 & Caesalpinia cacalaco & 4 & $\begin{array}{l}\text { Haematoxylum } \\
\text { campechianum }\end{array}$ & 3 & Guazuma ulmifolia & 0.24 \\
\hline $19^{\circ}$ & Diospyros nigra & 6 & Ehretia tinifolia & 4 & Caesalpinia cacalaco & 3 & Ficus yoponensis & 0.21 \\
\hline $20^{\circ}$ & $\begin{array}{l}\text { Roystonea } \\
\text { dunlapiana }\end{array}$ & 5 & Annona reticulata & 4 & Annona reticulata & 3 & Sabal mexicana & 0.19 \\
\hline
\end{tabular}

TABla 3. Tipos de usos de los árboles y número de especies por cada uno.

\begin{tabular}{ccc}
\hline Maderables & Extractivos & No extractivos \\
\hline construcción de casas (13) & artesanías (1) & cerca viva (19) \\
madera (16) & comestible (26) & conservación de agua (4) \\
muebles (5) & forraje (12) & cortina rompevientos (2) \\
tinturas (3) & insecticida (1) & ornamental (1) \\
& leña (14) & refugio de vida silvestre (12) \\
& maduración de mangos (1) & ritual (2) \\
& medicinal (11) & sombra (20) \\
& postes (13) & tutor (1) \\
& techos (2) & \\
& utensilios (15)
\end{tabular}


TABLA 4. Especies usadas para madera y construcción de casas, su uso y/o características.

\begin{tabular}{ll}
\hline Especie & $\begin{array}{l}\text { Uso y/o características de la } \\
\text { madera }\end{array}$ \\
\hline Acacia cochliacantha & horquetas para enramadas* \\
Attalea butyracea & vigas y alfardas* \\
Enterolobium cyclocarpum & resistente a la intemperie, tiene \\
& una sustancia que irrita los ojos \\
& al trabajarla \\
Diphysa robinioides & madera muy dura \\
Sabal mexicana & vigas y alfardas* \\
Salix humboldtiana & madera suave para albañilería \\
Tabebuia rosea & porque es fácil de clavar \\
Tabernaemontana alba & varengas* \\
Zuelania guidonia & fustes derechos y largos, ideal \\
& para vigas*
\end{tabular}

* en la construcción de casas: las vigas son los troncos que soportan la mayor carga de la casa, las alfardas se colocan en los techos para sostener las hojas de palma, las varengas se usan para hacer corrales de madera, las horquetas son las ramas que tienen forma de $Y$, las las enramadas son las construcciones ligeras y temporales que se ponen para dar sombra.

forraje para diferentes tipos de ganado, se aprovechan los frutos de Acacia cochliacantha, Annona reticulata, Attalea butyracea, Cordia dodecandra, Ficus aurea, Ficus yoponensis, Guazuma ulmifolia, Maclura tinctoria, Mangifera indica, Parmentiera aculeata; las hojas de Gliricidia sepium y Guazuma ulmifolia; y las flores de Ceiba pentandra. El uso como leña, principalmente para cocinar, se señaló como casi obvio, especialmente de aquellos árboles que no proveen otros beneficios; no obstante subrayaron las características de algunos de ellos (Tabla 5). Otro uso se da con las hojas de Gliricidia sepium cuando se mezclan con la fruta de mango (Mangifera indica) para acelerar su maduración. Para el uso medicinal las especies más señaladas fueron Pachira aquatica, Pterocarpus officinalis y Cecropia obtusifolia. La tabla 6 muestra las especies para las que se indicó la parte utilizada y el padecimiento para el cual se aplica.

Para postes se utilizan 13 especies, de las que sobresalen Maclura tinctoria por su durabilidad y Gliricidia
Tabla 5. Características de la leña de algunas especies usadas en la zona de estudio.

\begin{tabular}{ll}
\hline Especie & Características de la leña \\
\hline Acacia cochliacantha & muy buena para hacer pan \\
Ficus aurea & buena para horno de ladrillos \\
Gliricidia sepium & no echa humo \\
Guazuma ulmifolia & puede arder en verde \\
Maclura tinctoria & echa chispas y truena \\
Mangifera indica & mala para cocinar pues no deja brasa, \\
& buena para horno de ladrillos \\
\hline
\end{tabular}

TABla 6. Especies de árboles medicinales, los padecimientos que tratan y las partes que se utilizan.

\begin{tabular}{|c|c|}
\hline Especie & Padecimiento (parte utilizada) \\
\hline Bursera simaruba & sarampión, rubeola (hojas) \\
\hline Cecropia obtusifolia & $\begin{array}{l}\text { diabetes, dolor de huesos, reuma- } \\
\text { tismo (hojas) }\end{array}$ \\
\hline Cordia cf. diversifolia & dolor de rodillas (hojas) \\
\hline Guazuma ulmifolia & roña (corteza) \\
\hline Pachira aquatica & diabetes (fruto) \\
\hline Parmentiera aculeata & problemas de riñón (fruto) \\
\hline Psidium guajava & diarrea (hojas) \\
\hline Pterocarpus officinalis & diabetes, anemia (corteza) \\
\hline Randia sp. & picaduras de víbora (fruto) \\
\hline
\end{tabular}

sepium y Diphysa robinioides por ser buenas madrinas -postes más fuertes situados en las esquinas y donde se tensa el alambre. Para conservar la frescura de las casas tradicionales -escasas hoy día- suelen fabricar techos de hojas de palmas de Attalea butyracea (también registrada en la literatura como A. liebmannii) y de Sabal mexicana. Los utensilios más comunes son cabos para azadones o hachas. La tabla 7 muestra otros utensilios derivados de los árboles y las partes de las que se obtienen. La pólvora no se utiliza propiamente en la comunidad pero los informantes conocen ese uso porque les han comprado árboles para su extracción. 
TABLA 7. Ejemplos de utensilios que se obtienen de diferentes partes de los árboles y los materiales que los han ido sustituyendo.

\begin{tabular}{lll}
\hline Especie & Utensilios (parte utilizada) & Sustituto actual \\
\hline $\begin{array}{l}\text { Castilla elastica, Ficus } \\
\text { Ceiba aesculifolia, Ceiba }\end{array}$ & pelotas (látex) & juguetes de plástico \\
$\begin{array}{l}\text { pentandra } \\
\text { Cordia dodecandra }\end{array}$ & fibra para lavar loza (hojas) & relleno sintético de almo- \\
Ficus aurea & bateas (raíz), columpios (raíz) & hadas \\
& fibra de plástico \\
Ficus obtusifolia & mangas de hule (látex) & lavadoras, cuerdas de \\
Gliricidia sepium & horquetas para detener trojas -manojos- de & diversos materiales \\
Guazuma ulmifolia & ajonjolí (ramas bifurcadas y jóvenes) & mangas de plástico \\
palos de escoba, cortineros (ramas derechas y y & madera de pino de bajo \\
Manilkara zapota & jóvenes) & costo \\
chicle natural (látex) & yugos de yunta (raíz, tronco) & chicle artificial \\
Salix humboldtiana & escobas (ramas con hojas) & ya casi no se usa yunta, \\
\hline
\end{tabular}

\section{Usos no extractivos}

A los árboles también se les reconocen propiedades que permiten aprovecharlos y beneficiarse de ellos por su sola presencia. Las especies con más usos de este tipo y más mencionadas fueron Ehretia tinifolia, Pachira aquatica, Bursera simaruba, Maclura tinctoria y Ficus cotinifolia. Como cercas vivas se refirieron principalmente Gliricidia sepium, Bursera simaruba y Cordia dodecandra. Para la conservación del agua emplean Ehretia tinifolia, Ficus spp., Pachira aquatica y Salix humboldtiana. Como cortina rompevientos usan Casuarina equisetifolia y Ficus yoponensis. Como ornamental está Cassia fistula -especie exótica- por sus vistosas flores amarillas. Como refugio de vida silvestre se nombraron especies que proveen frutos carnosos y numerosos como Annona reticulata, Ficus spp., Maclura tinctoria y Manilkara zapota; árboles con oquedades en su estructura como Ehretia tinifolia y Ficus spp., y de troncos muy altos como Ceiba pentandra. Para uso ritual Ficus cotinifolia sirvió para rezar bajo su copa para pedir por lluvias cuando hubo un tiempo pro- longado de sequía y Cedrela odorata es considerado como árbol sagrado porque se dice que detrás de este árbol se escondió la virgen con el niño Jesús. Como especies para sombra más utilizadas están Pachira aquatica, Ficus aurea, Gliricidia sepium, Mangifera indica y Tabebuia rosea. Finalmente, el uso como tutor -el tronco sirve para dar sombra y crear las condiciones ambientales para soportar otras plantas de interés- señalaron a Ehretia tinifolia como tutor de Hylocereus undatus (pitaya - fruta comestible).

\section{Partes que se utilizan}

Gliricidia sepium, Ficus aurea y Guazuma ulmifolia son las especies de las que se usan más partes (Tabla 2). Se utiliza todo el tronco de $41 \%$ de las especies, lo que representa principalmente usos maderables; el fruto de $46 \%$, la mayoría para uso comestible o medicinal; las ramas de $32 \%$, usualmente para leña y postes; y las hojas de $19 \%$. En menor número de especies se usan las flores, semillas, corteza, brotes, raíz y látex. 


\section{Cambios en la comunidad a lo largo del tiempo}

Los informantes hablaron de la vida en las comunidades en las décadas de 1950 y 1960 aproximadamente. Los caminos eran de tierra y tardaban ocho horas en burro para llegar al Puerto de Veracruz (ciudad más cercana); hoy día tardan 30 minutos en auto. No tenían acceso a médicos ni medicamentos, vivían en casas de madera con techos de palma y estufas de leña, sobrevivían con lo que sembraban o colectaban en el bosque, cuya extensión era mucho mayor que la actual. El clima era más fresco, había más agua, las familias tenían muchos más miembros, no había acceso a escuelas. En los últimos 50 a 60 años el estilo de vida se ha transformado: hay mejores comunicaciones (i.e. carretera, teléfonos celulares, transporte público), hay acceso a clínicas y escuelas, las familias son más pequeñas, se ocupa menos tiempo en la preparación de comidas, hay entrada constante de otros productos envasados y procesados, hay electricidad, ha habido migración al extranjero o a las ciudades y la vegetación se ha reducido mucho. Estos cambios también trajeron nuevos materiales u objetos que sustituyeron parcial o totalmente a los que tradicionalmente se obtenían de los árboles (Tabla 7).

\section{DISCUSIÓN}

Los resultados de las encuestas y la búsqueda de informantes a través de la bola de nieve, muestran un fuerte sesgo de género entre los informantes; hay menos mujeres dueñas de terrenos que hombres, denotando que la ganadería es un gremio mayoritariamente masculino. No obstante $26 \%$ de mujeres informantes en este estudio sobrepasa 6\% encontrado por Vilaboa y Díaz (2009), si bien estos autores manejaron una muestra diez veces mayor que la de este estudio. El número de especies registradas en este trabajo (68) es menor a lo encontrado sobre todo en zonas con población indígena (Casas et al., 1994; Levy et al., 2002; Rico et al., 1991) o en trabajos extensos de etnobotánica (Toledo et al., 1995), pero similar a lo encontrado por Muñoz (2006), que estudió el conocimiento de árboles que tenían los ganaderos en Costa Rica.

\section{Usos}

Se mencionaron muchos más usos que los meramente relacionados con la ganadería (postería, cerca viva, forraje y sombra), mostrando que los beneficios que se obtienen de los árboles no solo son para esta actividad económica sino que abarcan otras necesidades de la vida cotidiana. La categoría de usos maderables representa $18 \%$ de los tipos de utilización; no obstante, al utilizar todo el árbol se ponen en detrimento los usos extractivos y no extractivos. Por ejemplo, hay especies de plantas comestibles y medicinales como bejucos y herbáceas que crecen sobre los árboles y que se pierden como consecuencia de la tala. El uso para tinta no es muy habitual en la zona, pero cabe mencionar el caso de Brasil donde la especie Caesalpinia echinata fue llevada casi a la extinción para la extracción de tinta en tiempos de la colonización (Bolzani y Barreiro, 2006). Por ello, en especial para los usos maderables, es indispensable la planificación y la resiembra para evitar la desaparición de las especies que conlleva pérdidas irreparables del acervo genético, así como para lograr un manejo más sustentable.

En cuanto a los usos extractivos, el uso comestible fue el más recurrente ( $c$ a. 38\% de las especies). Sin embargo ha cambiado el consumo de frutos silvestres y se ha ido reemplazando por otras frutas cultivadas o que llegan desde otros lugares. Por esta razón posiblemente los árboles ya no son una fuente tan importante de alimento aunque antiguamente sí lo fueron. Por ejemplo, el fruto del coyol (Attalea butyracea) era consumido para hacer tortillas y atoles pero este conocimiento y costumbre ya solo queda en las personas mayores (González et al., 2012a). Las especies de árboles que actualmente se consumen son usualmente frutales que no pertenecen al bosque nativo como el mango, el aguacate y el tamarindo; o bien, ya solo se consumen algunas variedades que tampoco son nativas del lugar, como el caso de las guayabas que vienen de fuera. En un vivero local de un grupo de mujeres de la comunidad, las plantas más buscadas por la propia comunidad son estos frutales. Por lo tanto, el uso comestible no sería tan preponderante si solo se consideran las especies nativas; los árboles frutales probablemente no requieren medidas de conservación pero los árboles de bosque nativo 
sí las pueden necesitar. En este sentido, Lascurain et al. (2010) proponen recuperar el consumo de frutos nativos como estrategia para aumentar la seguridad alimentaria rural y ayudar a conservar las especies locales. El uso medicinal de los árboles sigue siendo importante en la zona (Escamilla, 2013) y representa $16 \%$ de las especies de este estudio. Las tres especies más usadas son para tratamiento de la diabetes (Tabla 6), una enfermedad relativamente reciente en las zonas rurales y que se ha incrementado por el consumo de comida de baja calidad nutricional (Jiménez, 2007), revelando un cambio radical en los hábitos de la población en detrimento de su salud. Para la construcción de techos, las especies usadas se limitan a dos tipos de palmas, coincidiendo con González et al. (2012a). El uso de una tercera palma de humedales, Roystonea dunlapiana, es más limitado porque es más escasa, crece lentamente, ha sido muy afectada por la disminución de humedales y está protegida legalmente (González et al., 2012a). Asimismo, las palmas se han sustituido por materiales como lámina o cemento, aunque los precios sean mayores y guarden más calor dentro de las casas (González et al., 2012a). El uso para leña en la zona es muy común; en México y otras zonas rurales a nivel mundial la leña aporta $80 \%$ de la energía usada en el campo (Abbot et al., 1997, Masera et al., 2006). La identificación de las características de la leña por los informantes de este estudio es similar a los resultados de Abbot et al. (1997) en el centro sur de África, quienes también aprecian propiedades como la duración de la brasa.

Dentro de los usos no extractivos de los árboles sorprende que no se haya mencionado la producción de miel. No hubo menciones de que se haya realizado esta actividad en la zona a pesar de que varias especies -e.g. Bursera simaruba, Persea americana- tienen ese potencial (Montoy, 2010). El uso para sombra, en especial de Mangifera indica y Ficus spp. coinciden con las sombras consideradas como "buenas" encontradas por Muñoz (2006) en Costa Rica. Ese mismo autor recalca que cuando una sombra es demasiado densa tampoco gusta tanto a los ganaderos pues impide el crecimiento del pasto. Este es uno de los factores limitantes para la adopción de sistemas silvopasto- riles, que combinan la producción de ganado con el aprovechamiento sistemático de los árboles (Mahecha, 2003).

Otros usos no extractivos que resultaron de esta investigación tienen que ver con los servicios ecosistémicos (sensu Millenium Ecosystem Assessment, 2005): de aprovisionamiento como la conservación del agua; de regulación como el control de la erosión por la protección del viento con las cercas vivas; de soporte de la biodiversidad como el hábitat de fauna silvestre; y culturales como el uso ritual de algunas especies. Esta valorización no material por parte de los informantes puede favorecer la conservación de los árboles (Svorc y Oliveira, 2012).

\section{Importancia cultural}

El índice de importancia cultural (IIC) mostró las especies más significativas para este grupo de informantes al momento de la entrevista, es decir, si se preguntara a otros informantes con otros intereses, las especies serían distintas. Asimismo, preguntar a los mismos informantes hace 50 años hubiera dado otro resultado. Esta metodología permitió registrar los árboles más apreciados por los ganaderos de hoy en día, no obstante se encontró que las especies con valor más alto no necesariamente corresponden a las más usadas en la actividad ganadera. Al calcular el IIC, las especies que son únicas para un uso en particular ganan un puntaje muy alto, el valor total de uso ( vut $_{\mathrm{z}}$ ) se dispara frente a los otros dos parámetros (intensidad de uso y frecuencia de mención) del indicador. Por ejemplo en el caso de Ehretia tinifolia -es el único usado como tutor y de los pocos mencionados para la conservación del agua$\mathrm{y}$ en principio se encuentra en los primeros cinco lugares de importancia cultural, pero si no se considera el vut, pasa hasta el lugar 16 de la lista. Cuando una especie es la única para un uso, el índice considera que tiene una importancia cultural más alta porque no tiene sustitutos. Es interesante el caso de Cedrela odorata que tiene el IIC más alto de todas las especies; aunque no sobresale ni en el número de usos ni en el número de partes que se utilizan (Tabla 2); el resultado del índice logra reflejar que es uno de los árboles favoritos en la comunidad como fue indicado por los informantes. 


\section{Vegetación}

Los resultados muestran la importancia de las especies de vegetación secundaria, que es similar al estudio de Chazdon y Coe (1999), quienes hallaron que 70\% de las especies leñosas muestreadas en su estudio eran de este tipo de vegetación. Ello indica que aunque haya un fuerte problema de perturbación del bosque primario, la vegetación secundaria provee también múltiples beneficios y es de interés para los usuarios locales. La vegetación de humedales, principalmente de selva inundable, es importante en la zona. El trabajo de Infante et al. (2014) demuestra la importancia de conservar este tipo de ecosistemas para beneficio de la sociedad local, lo que hace urgente tomar acciones que conserven los humedales funcionales para seguir suministrando servicios ecosistémicos, como aprovisionamiento de agua, contención de inundaciones, entre otros.

Algunas especies exóticas como Azadirachta indica, Cocos nucifera, Gmelina arborea y Casuarina equisetifolia fueron introducidas en la zona. Algunas de ellas han sido promovidas para aumentar la productividad o dar alternativas económicas a los productores, sin embargo ha faltado mayor atención a posibles consecuencias de la introducción de estas especies como plagas sin enemigos naturales, especies invasoras y desequilibrio en los procesos ecológicos (Vázquez y Batis, 1996). Cabe decir que en la región no se mencionó ningún problema en este sentido. Por otra parte, aunque Mangifera indica es una especie exótica, lleva más de 60 años en la región (Escamilla, 2013) y ya es uno de los árboles con mayor número de usos. En contraste, Azadirachta indica se introdujo hace pocos años y aunque tiene muchos usos en India, su lugar de origen (Biswas et al., 1995), en la zona solamente se utiliza como insecticida. Esto sugiere que el conocimiento local se va enriqueciendo con la experiencia empírica sobre el uso de los recursos arbóreos, incluso en poblaciones no indígenas.

\section{Cambios a lo largo del tiempo}

La información sobre el estilo de vida en las décadas de 1950 y 1960 coincide con las fechas en que el uso de los árboles era más intensivo y se había desmontado $32 \%$ de los terrenos de este estudio. Esto podría mostrar que había una mayor cantidad de bosque y se hacía un uso más diverso de los árboles, lo cual presupone un amplio conocimiento sobre los mismos. No obstante, algunos autores han demostrado que la pérdida de árboles no necesariamente motiva a los locales a sembrar más (Kishor y Mitchell, 2004), especialmente si hay sustitutos para su uso (Gordon et al., 2003), como en el caso de este estudio. Moreno-Casasola y Paradowska (2009) encontraron que en los bosques tropicales caducifolios de las dunas, hay especies que la gente reconoce y aprecia, pero no están dispuestos a sembrar aunque ya no haya, debido a que se propagan solos (aunque después no se cuiden las condiciones para favorecer su desarrollo) o no hay seguridad de quién utilizará esos individuos en el futuro.

El mecanismo de búsqueda de informantes por bola de nieve muestra que en la propia zona no se reconoce que la gente más joven (menor de 40 años) se dedique a la actividad ganadera o tenga muchos conocimientos sobre el uso de los árboles. En los resultados se nota que aunque los productores actuales poseen conocimientos sobre el uso de los árboles, ya no lo están utilizando porque ya no lo necesitan (como el caso de Attalea butyracea) o porque ya son muy escasos (como el caso de Zuelania guidonia). Ante esto, parece probable una pérdida del conocimiento tradicional, pues difícilmente pasará a la siguiente generación (Reyes, 2009; Marín, 2013).

\section{CONCLUSIONES}

Los ganaderos entrevistados poseen un amplio conocimiento sobre las especies arbóreas y sus usos. El presente trabajo es una contribución importante para la documentación de este acervo de conocimiento etnobotánico ante su probable pérdida en el futuro próximo. El tipo, el uso, la cantidad y la salud de los árboles son un reflejo del modo de vida de los habitantes de la zona, incluyendo sus necesidades, preferencias e influencias. Si bien el estilo de vida actual tiene beneficios para la población, como mayor comodidad o mayor acceso a información, también tienen repercusiones como nuevas enfermedades, hábitos de ali- 
mentación menos saludables y desapropiación de los recursos naturales. La sustitución de materiales naturales por la utilización de plásticos por ejemplo, ha disminuido la presión hacia los árboles pero por otro lado ha aumentado la cantidad de residuos no biodegradables, entre otros efectos secundarios.

La pérdida de la vegetación arbórea y de sus servicios ecosistémicos es un problema complejo que exige la renovación de la actividad ganadera con acciones en favor de la sustentabilidad. El trabajo de incrementar el arbolado en potreros es una vía importante para mantener la conectividad, ayudar a la conservación de la biodiversidad y el germoplasma y mantener tradiciones. Esto se vuelve primordial al considerar la gran extensión de tierra dedicada a la ganadería en Veracruz, así como por el bienestar de las localidades rurales usuarias de estos recursos. La configuración del paisaje depende en gran medida de las decisiones individuales de cada propietario de terrenos, haciendo urgente el trabajo con este gremio para favorecer la conservación de sus recursos. En este sentido, ya existen incentivos de la Sagarpa (Secretaria de Agricultura, Ganadería, Desarrollo Rural, Pesca y Alimentación) que impulsan el mantenimiento de arbolado en los potreros, aunque pocos ganaderos hacen uso de estas posibilidades. Por otro lado se requiere trabajo de educación ambiental e incentivos para la siembra y sobre todo para el cuidado de los árboles sembrados, no solo en plantaciones sino bajo las condiciones actuales de actividad agropecuaria. Algunas acciones son promover un mayor uso de los árboles nativos en los potreros, tanto en sistemas silvopastoriles como en cercas vivas; impulsar plantaciones para producción de leña; resembrar especies nativas para recuperación del acervo genético; valorizar los recursos comestibles silvestres; mantener las zonas riparias forestadas y mantener y manejar los árboles aislados dentro de los potreros. Para evitar la pérdida del conocimiento de usos tradicionales es fundamental involucrar a las generaciones más jóvenes, que próximamente estarán encargadas del manejo de su territorio y los recursos naturales. Asimismo, se puede enriquecer este trabajo en el futuro estudiando más a fondo la abundancia, composición y estado sucesional de las especies de árboles presentes en la actualidad, así como investigar de forma cuantitativa los cambios en la necesidad de uso de materiales derivados de los árboles. En suma, la conservación de los árboles, su conocimiento y los servicios ecosistémicos que proveen requiere una población rural más activa y formada, que conozca sus recursos y tenga más bases de conocimiento para tomar decisiones y para ayudar a moldear las políticas que regulan su territorio (Lazos-Ruíz et al., 2013).

\section{RECONOCIMIENTOS}

A todos los entrevistados y sus familias, Abraham Juárez (†), Maricruz Peredo (†), Carlos Ramírez, Rubén Lazos y los revisores anónimos cuyos comentarios ayudaron a mejorar este artículo. Esta investigación fue realizada con fondos de OIMT del proyecto RED-PD 045/11 Rev.2 (M) y la beca doctoral otorgada por Conacyt a la primera autora (no. 208529).

\section{REFERENCIAS}

Abbot, P., J. Lowore, C. Khofi y M. Werren. 1997. Defining firewood quality: a comparison of quantitative and rapid appraisal techniques to evaluate firewood species from a Southern Africa Savanna. Biomass and Bioenergy 12(6):429-437.

Avendaño, S. 1998. Bombacaceae. Flora de Veracruz. Instituto de Ecología, A.C. Xalapa. 42 p.

Avendaño, S. e I. Acosta. 2000. Plantas utilizadas como cercas vivas en el estado de Veracruz. Madera y Bosques 6(1):5571.

Ávila, D., O. Rosas, L. Tarango, J. Martínez y E. Santoyo. 2011. Conocimiento, uso y valor cultural de seis presas del jaguar (Panthera onca) y su relación con éste, en San Nicolás de los Montes, San Luis Potosí, México. Revista Mexicana de Biodiversidad 82:1020-1028.

Bellefontaine, R., S. Petit, M. Pain-Orcet, P. Deleporte, y J. Bertault. 2002. Los árboles fuera del bosque. Guía FAO Conservación 35.237 p.

Betancourt, K., M. Ibrahim, C. Harvey y B. Vargas. 2003. Efecto de la cobertura arbórea sobre el comportamiento animal en fincas ganaderas de doble propósito en Mati- 
guás, Matagalpa, Nicaragua. Agroforestería de las Américas 10(39-40):47-51.

Biswas, S., P. Singh y S. Chandra. 1995. Neem (Azadirachta indica A. Juss) a versatile multipurpose tree. The Indian Forester 121(11):1057-1062.

Bolzani, C. y E. Barreiro. 2006. Os productos naturais e a química medicinal moderna. Química Nova 29(2):326337.

Bravo, L., O. Doode, A. Castellanos e I. Espejel. 2010. Políticas rurales y pérdida de cobertura vegetal. Elementos para reformular instrumentos de fomento agropecuario relacionados con la apertura de praderas ganaderas en el noroeste de México. Región y Sociedad 22(48):3-35.

Casas, A. 2001. Silvicultura y domesticación de plantas en Mesoamérica. In: B. Rendón, S. Rebollar, J. Caballero y M. Martínez, eds. Plantas, Cultura y Sociedad. UAMSemarnat. México, D.F. p:123-158.

Casas, A., J. Viveros y J. Caballero. 1994. Etnobotánica mixteca: sociedad, cultura y recursos naturales en la montaña de Guerrero. Consejo Nacional de la Cultura y las Artes e Instituto Nacional Indigenista. México, D.F. 366 p.

Castillo, G. y M. Medina. 2005. Árboles y arbustos de la Reserva Natural de La Mancha, Veracruz. Instituto de Ecología, A.C. Xalapa. 144 p.

Castillo, G., y A. Travieso. 2006. La Flora. In: P. Moreno-Casasola, ed. Entornos veracruzanos: la costa de La Mancha. Instituto de Ecología, A.C. Xalapa. p:171-204.

Céspedes, S. y E. Moreno. 2010. Estimación del valor de la pérdida de recurso forestal y su relación con la reforestación en las entidades federativas de México. Investigación ambiental. Ciencia y política pública 2(2):5-13.

Chazdon, R. y F. Coe. 1999. Ethnobotany of woody species in second-growth, old-growth, and selectively logged forests of northeastern Costa Rica. Conservation Biology 13(6):1312-1322.

Conafor (Comisión Nacional Forestal). 2012. Inventario nacional forestal y de suelos. Informe 2004-2009. ConaforSemarnat. Zapopan. 212 p.

Davis, C., H. Gallardo y K. Lachlan. 2010. Talking straight about communication research methods. Kendall Hunt Publishing Co. Dubuque. 448 p.
Escamilla, B. 2013. Valoración del servicio ambiental de provisión de los recursos naturales de un potrero derivado de selva-palmar inundable, en Jamapa, Veracruz. Tesis de Maestría. Universidad Autónoma de Baja California. Ensenada. 133 p.

Esquivel, H., M. Ibrahim, C. Harvey, C. Villanueva, T. Benjamin y F. Sinclair. 2003. Árboles dispersos en potreros de fincas ganaderas en un ecosistema seco de Costa Rica. Agroforestería en las Américas 10(39-40):24-29.

FAO (Organización de las Naciones Unidas para la Agricultura y la Alimentación). 2010. Evaluación de los recursos forestales mundiales 2010. Estudio FAO Montes 163. Roma. 346 p.

Gómez, H., D. Galdámez, F. Guevara, A. Ley y R. Pinto. 2013. Evaluación de áreas ganaderas en la zona de amortiguamiento de una reserva natural en Chiapas, México. Información Técnica Económica Agraria 109(1):69-85.

Gómez-Pompa, A. 1987. On Maya Silviculture. Mexican Studies/Estudios Mexicanos 3(1):1-17.

González, R., P. Moreno-Casasola, R. Orellana y A. Castillo. 2012a. Palm use and social values in rural communities on the coastal plains of Veracruz, Mexico. Environment, Development and Sustainability 14(4):541-555.

González, R., P. Moreno-Casasola, R. Orellana y A. Castillo. 2012b. Traditional wetland palm uses in construction and cooking in Veracruz, Gulf of Mexico. Indian Journal of Traditional Knowledge 11(3):408-413.

Gordon, J., A. Barrance y K. Schreckenberg. 2003. Are rare species useful species? Obstacles to the conservation of tree diversity in the dry forest zone agro-ecosystems of Mesoamerica. Global Ecology and Biogeography 12(1):13-19.

Guevara, S., J. Laborde y G. Sánchez-Ríos. 2005. Los árboles que la selva dejó atrás. Interciencia 30(10):595-601.

Guevara, S. y P. Moreno-Casasola. 2008. El dilema de los recursos naturales: La ganadería en el Trópico de México. Guaraguao 29:9-23.

Ibarra, G., G. Cornejo-Tenorio, N. González-Castañeda, E. Piedra-Malagón y A. Luna. 2012. El género Ficus L. (Moraceae) en México. Botanical Sciences 90(4):389-452.

Inegi (Instituto Nacional de Estadística, Geografía e Informática). 2009. Información por municipio. Prontuario de 
información geográfica municipal de los Estados Unidos Mexicanos. Jamapa, Veracruz de Ignacio de la Llave, en línea en http://www3.inegi.org.mx/sistemas/mexicocifras/datos-geograficos/30/30090.pdf. Consultada en febrero 2014.

Inegi (Instituto Nacional de Estadística, Geografía e Informática). 2010. Principales Resultados del Censo de Población y Vivienda 2010, en línea en http://www.inegi.gob.mx/ prod_serv/contenidos/espanol/bvinegi/productos/censos/ poblacion/2010/princi_result/cpv2010_principales_resultadosI.pdf . Consultada en febrero 2014.

Infante, D., P. Moreno-Casasola y C. Madero. 2014. ¿Pachira aquatica, un indicador del límite del manglar? Revista Mexicana de Biodiversidad 85:143-160.

Jimenez, A. 2007. Obesidad, diabetes y pobreza: costos e implicaciones. Ciencias 58(2), en línea en http://www.revistaciencia.amc.edu.mx/. Consultada en febrero 2014.

Kishor, K. y C. Mitchell. 2004. Do socio-psychological factors matter in agroforestry planning? Lessons from smallholder traditional agroforestry systems. Small-scale Forest Economics, Management and Policy 3(2):239-255.

Landgrave, R. y P. Moreno-Casasola. 2012. Evaluación cuantitativa de la pérdida de humedales en México. Investigación ambiental. Ciencia y política pública 4(1):19-35.

Lascurain, M., S. Avendaño, S. del Amo y A. Niembro. 2010. Guía de frutos comestibles en Veracruz. Conafor-Conacyt. México. 144 p.

Lazos-Ruíz, A., P. Moreno-Casasola y E. Galante. 2013. Empresa Rural Verde: desarrollando criterios de sustentabilidad con la comunidad rural. Revista Forum de Sostenibilidad 6:3-16.

Letts, L., S. Wilkins, M. Law, D. Stewart, J. Bosch y M. Westmorland. 2007. Guidelines for critical review form: qualitative studies. McMaster University. Ontario. 12 p.

Levy, S., J. Aguirre, M. Martínez y A. Durán. 2002. Caracterización del uso tradicional de la flora espontánea en la comunidad Lacandona de Lacanhá, Chiapas, México. Interciencia 27(10):512-520.

Lindenmayer, D., W. Laurance, y J. Franklin. 2012. Global decline in large old trees. Science 338(6112):1305-1306.
López-Austin, A. 1997. El árbol cósmico en la tradición mesoamericana. Monografías del Jardín Botánico de Córdoba $5: 85-98$

Mahecha, L. 2003. Importancia de los sistemas silvopastoriles y principales limitantes para su implementación en la ganadería colombiana. Revista Colombiana de Ciencias Pecuarias 16(1):11-18.

Marín, J. 2013. Balance neto de carbono en suelos de humedales costeros de agua dulce: implicaciones ecológicas y sociales. Tesis Doctoral. Universidad Veracruzana. Xalapa. $155 \mathrm{p}$.

Masera, O., R. Díaz y V. Berrueta. 2006. Programa para el uso sustentable de la leña en México: de la construcción de estufas a la apropiación de la tecnología. Revista Digital Entorno TCSD 03-05.

Millenium Ecosystem Assessment. 2005. Ecosystems and Human Well-being: Wetlands and Water. World Resources Institute. Washington, D.C. 68 p.

Montoy, L. 2010. Estudio apibotánico para un mejor aprovechamiento de los recursos naturales en la región de las montañas, Veracruz. Tesis de grado para Ingeniero Agrónomo. Instituto Tecnológico de Chiná. Campeche.70 p.

Moreno, A. 2011. Efectos ambientales del Programa Nacional de Desmontes, México, 1972-1982. Tesis de posgrado para Maestría en Ciencias Ambientales. Universidad Autónoma de San Luis Potosí y Universidad de Ciencias Aplicadas de Colonia. Colonia. 119 p.

Moreno-Casasola, P. y D. Infante. 2009. Manual del Manglar y Selvas Inundables. Instituto de Ecología, A.C., Conafor, OIMT. Xalapa. 101 p.

Moreno-Casasola, P. y K. Paradowska. 2009. Especies útiles de la selva baja caducifolia en las dunas costeras del centro de Veracruz. Madera y Bosques 15(3):21-44.

Muñoz, D. 2006. Conocimiento local de la cobertura arbórea en sistemas de producción ganadera en dos localidades de Costa Rica. Revista de Ciencias Agrícolas 23 (1):155170 .

Nash, D. y N. Moreno. 1981. Boraginaceae. Flora de Veracruz. Instituto de Ecología A.C. Xalapa. 307 p.

Niembro, A. 2001. Las diásporas de los árboles y arbustos nativos en México: posibilidades y limitaciones de uso en pro- 
gramas de reforestación y desarrollo agroforestal. Madera y Bosques 7(2): 3-11.

Niembro, A., M. Vázquez y O. Sánchez. 2010. Árboles de Veracruz. 100 especies para la reforestación estratégica. Gobierno de Veracruz. Xalapa. 253 p.

Pennington, T. y J. Sarukhán. 2005. Árboles tropicales de México. UNAM, Fondo de Cultura Económica. México, D.F. 523 p.

Quero, H. 1994. Palmae. Flora de Veracruz. Instituto de Ecología A.C. Xalapa. 118 p.

Reyes, V. 2009. Conocimiento ecológico tradicional para la conservación: dinámicas y conflictos. Papeles 107:39-55.

Rico, V., A. Chemás y S. Mandujano. 1991. Uses of tropical deciduous forest species by the Yucatecan Maya. Agroforestry Systems 14(2):149-161.

Rzedowski, J. 2006. Vegetación de México. Comisión Nacional para el Conocimiento y Uso de la Biodiversidad. México, D.F. 505 p.

Sedarpa (Secretaría de Desarrollo Agropecuario Rural y Pesca). 2012. Programa veracruzano de agricultura, ganadería, forestal, pesca y alimentación 2005-2010, en línea en http://www.veracruz.gob.mx/finanzas/files/2012/01/ tf07-ps-sedarpa.pdf. Consultada en septiembre 2015.

Sefiplan (Secretaría de Finanzas y Planeación del Estado de Veracruz). 2013. Cuadernillos Municipales. Jamapa. Sistema de Información Municipal. Veracruz. 10 p.

Svorc, R. y R. Oliveira. 2012. Uma dimensão cultural da paisagem: biogeografía e história ambiental das figueiras centenárias da Mata Atlântica. GEOUSP-espaço e tempo 32:140-160.

Tarrés, M. L. 2004. Observar, escuchar y comprender sobre la tradición cualitativa en la investigación social. Flacso. México, D.F. 407 p.

Toledo, V. 1990. El proceso de ganaderización y la destrucción ecológica de México. In: E. Leff, coord. Medio ambiente y desarrollo en México. UNAM-CIIH-Porrúa. México, D.F. p:191-228.
Toledo, V., A. Batis, R. Becerra, E. Martínez y C. Ramos. 1995. La selva útil: etnobotánica cuantitativa de los grupos indígenas del trópico húmedo de México. Interciencia 20(4):177-187.

Travieso-Bello, A. y P. Moreno-Casasola. 2011. Sustentabilidad de la ganadería bovina: el caso de la costa de Actopan, Veracruz, México. In: M. Sánchez, A. Contreras y E. Kauffer, coord. La encrucijada en México rural. Contrastes regionales en un mundo desigual. Asociación Mexicana de Estudios Rurales. México, D.F. p:291-316.

Turner, N. 1988. The importance of a rose. Evaluating the cultural significance of plants in Thompson and Lilloet Interior Salish. American Anthropologist 90:272-290.

UNAM (Universidad Nacional Autónoma de México). 2009. Biblioteca digital de la medicina tradicional mexicana. En línea en http://www.medicinatradicionalmexicana.unam. mx. Consultado en febrero 2014.

Vázquez, C. y A. Batis. 1996. Adopción de árboles nativos valiosos para la restauración ecológica y la reforestación. Boletín de la Sociedad Botánica de México 58:75-84.

Vilaboa, J. y P. Díaz. 2009. Caracterización socioeconómica y tecnológica de los sistemas ganaderos en siete municipios del estado de Veracruz, México. Zootecnia Tropical 27(4):427-436.

Villa, A., M. Nava, S. López, S. Vargas, E. Ortega y F. Gallardo. 2009. Utilización del guácimo (Guazuma ulmifolia Lam.) como fuente de forraje en la ganadería bovina extensiva del trópico mexicano. Tropical and Subtropical Agroecosystems 10(2):253-261.

Manuscrito recibido el 12 de junio de 2014. Aceptado el 13 de octubre de 2015.

Este documento se debe citar como:

Lazos-Ruíz, A., P. Moreno-Casasola, S. Guevara S., C. Gallardo y E. Galante. 2016. El uso de los árboles en Jamapa, tradiciones en un territorio deforestado. Madera y Bosques 22(1):17-36. 
Anexo 1. Entrevista semiestructurada utilizada en el municipio de Jamapa.

I. Datos del entrevistado

Nombre

Edad

Comunidad

Ocupación

¿Su terreno se inunda?

II. Árboles

¿Qué árboles conoce y utiliza?

¿Para qué?

¿Qué partes del árbol usa?

III. Cambios en el tiempo

¿Hace cuánto tiempo fue desmontado su terreno?

¿Cómo era la vida antes?

¿Cómo ha cambiado esta comunidad desde que usted se acuerda?

¿Los árboles tenían otros usos? 